\title{
Segmentation of Densely Populated Cell Nuclei from Confocal Image Stacks using 3D Non-parametric Shape Priors
}

\author{
Lee-Ling S. Ong ${ }^{1}$, Mengmeng Wang ${ }^{2}$, Justin Dauwels ${ }^{2}$ and H. Harry Asada ${ }^{1,3}$
}

\begin{abstract}
An approach to jointly estimate $3 D$ shapes and poses of stained nuclei from confocal microscopy images, using statistical prior information, is presented. Extracting nuclei boundaries from our experimental images of cell migration is challenging due to clustered nuclei and variations in their shapes. This issue is formulated as a maximum a posteriori estimation problem. By incorporating statistical prior models of $3 D$ nuclei shapes into level set functions, the active contour evolutions applied on the images is constrained. A $3 D$ alignment algorithm is developed to build the training databases and to match contours obtained from the images to them. To address the issue of aligning the model over multiple clustered nuclei, a watershed-like technique is used to detect and separate clustered regions prior to active contour evolution. Our method is tested on confocal images of endothelial cells in microfluidic devices, compared with existing approaches.
\end{abstract}

\section{INTRODUCTION}

To quantify cell morphology and motion for cell migration studies, we would first identify or segment individual cells or nuclei. Automatic segmentation of cellular objects is a more efficient and unbiased approach compared to manual or semi-automatic methods. The majority of nuclei segmentation techniques are applied to segment nuclei on a twodimensional $(2 D)$ surface [1], [2].

Here, we present a methodology to segment stained nuclei from three-dimensional $(3 D)$ confocal images acquired from experiments in microfluidic devices [3]. Figure 1 shows an example of the images typically acquired. This image comprises of nuclei in gel area and non-gel areas as well as densely clustered nuclei at the gel boundaries (indicated by the red ellipse). It is a challenge to accurately separate clustered nuclei shapes automatically [4]. Although watershed techniques can be used to separate clustered $3 D$ nuclei [5], this approach often fails to identify the whole nuclei shape due to oversegmentation or undersegmentation.

When biologists manually segment confocal images, they often apply some prior information about nuclei shape. It is desirable to develop automatic analysis software which incorporates such information. Active contours is a popular approach to enforce shape constraints [6], [7]. Templates are created to capture shape variability. A number of algorithms apply templates directly by creating parametric point

\footnotetext{
${ }^{1}$ L.L.S.Ong and H.H. Asada are with Singapore-MIT Alliance for Research and Technology, Singapore. sharon.ong at smart.mit.edu

${ }^{2} \mathrm{M}$. Wang and J. Dauwels are with the School of Electrical and Electronic Engineering, Nanyang Technological University, Singapore. jdauwels at ntu.edu.sg

${ }^{3}$ H.H. Asada is also with the Department of Mechanical Engineering, MIT, Cambridge, MA, USA. asada at mit.edu
}

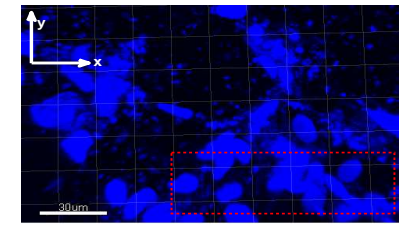

(a) Confocal Image in XY plane

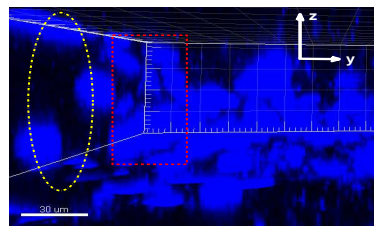

(b) Confocal Image in $\mathrm{YZ}$ plane
Fig. 1. 3D confocal stack of stained nuclei images (blue) at different axes, visualized with IMARIS (Bitplane), are acquired from our experimental device. (a) shows the cells in the gel area. The red rectangle in both figures indicate nuclei at the $3 \mathrm{D}$ non-gel to gel interface where cells clump together. The yellow ellipse in (b) indicates the area without gel. Nuclei in this region attach to the top or bottom of the device. (Scale bar $=30 \mu \mathrm{m}$.)

distributions or spline models to describe the segmenting curve [8]. However, the success of these approaches is dependant on the quality and diversity of the templates [6]. We represent the training shapes as level-set distance functions [6]. We update the level-set obtained from images, with statistical prior models in a nonparametric multivariate kernel density estimation framework [9].

Next, we derive an alignment algorithm to match the $3 D$ image-driven level set with the $3 D$ training database. The nuclei pose (location, orientation and scale) is estimated when we align the image. To avoid matching prior models for a single nuclei with multiple nuclei, we use a watershed-like technique to detect and separate connected nuclei. We then convert the separated nuclei contours to level set functions and update the functions separately with the prior models.

Furthermore, our methodology accounts for variations in nuclei shape due to cell location (e.g whether the cell is in the gel or non-gel environments) by creating multiple shape databases. To the best of our knowledge, there is no existing work which jointly estimates $3 D$ nuclei poses and shape using statistical prior models. We test our method on confocal images of endothelial cells in microfluidic devices, comparing our approach with existing ones. This work is also applicable to segment different types of $3 D$ images such as MRI data.

\section{METHOD}

Figure 2 illustrates how we incorporate prior models to jointly estimate nuclei pose $\mathbf{p}$ and curve shape $\mathbf{C}$. We first evolve the level set function without any shape priors to obtain the initial curve $\mathbf{C}$. We then check for multiple nuclei using the $3 D$ watershed-like technique described. For each labeled nuclei, we compute the initial pose $\mathbf{p}$ and align 


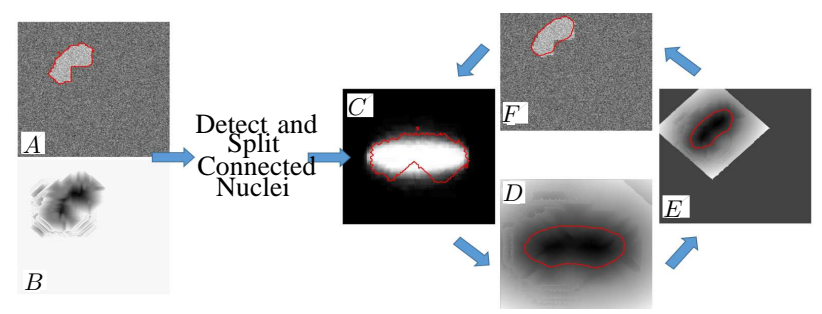

Fig. 2. Flow diagram to estimate nuclei shape and pose with prior models. From the example nuclei image in (A), we evolve the level set function without prior information to obtain $\psi$ in (B). Using a watershedlike algorithm, we split connected nuclei and create new level set functions for each separated nuclei. In (C), we determine the nuclei pose by aligning the shape $\mathbf{C}$ (red curve) to the training shapes. The level set estimate $\left(\psi_{\tilde{C}}\right)$, obtained by transposing $\psi_{\mathbf{C}}$ from (B), is updated with $n$ shape prior models, to obtain (D). In (E), we transpose the updated level set in(D) to its original pose. $(\mathrm{F})$ is the updated curve evolved with the image force. The new pose is then updated. Steps $(\mathrm{C})$ to $(\mathrm{F})$ are iterated until convergence.

its curve $\mathbf{C}$ with respect to the template to obtain $\tilde{C}$ and underlying level set. The aligned level set is updated with shape prior models. We then transpose the aligned level set back its original frame using the initial pose. We then evolve this levelset with the image driven force and determine the new pose.

\section{A. Estimation of shape and pose with prior models}

We segment the cell by obtaining its candidate boundary curve $\mathbf{C}$, through minimizing the energy functional:

$$
E(\mathbf{C})=-\log p(\text { data } \mid \mathbf{C})-\log p_{\mathbf{C}}(\mathbf{C}),
$$

where $p_{\mathbf{C}}(\mathbf{C})$ is the prior density of the curve $\mathbf{C}$, which incorporates nuclei shape models.

To build a training database of curves $\mathbf{C}_{i}, \ldots, \mathbf{C}_{n}$, we apply a similarity transform, containing pose parameters to align the curves. We denote $\mathbf{p}$ as the estimated pose of $C$ and $\tilde{C}=T[\mathbf{p}] C$ as the shape obtained by transforming every point on the curve. We estimate the density $p_{\mathbf{C}}(\mathbf{C})$ of any segmenting curve as $p_{\mathbf{C}}(\mathbf{C}) \approx p_{\tilde{C}}(T[\mathbf{p}] \mathbf{C})$.

We then update the transformed segmenting curve $\tilde{C}$ with the training data through a Parzen density representation (using Gaussian kernels) $p_{\tilde{C}}($.$) which is defined in [9] as:$

$$
p_{\tilde{C}}(\tilde{C})=\frac{1}{n} \sum_{i=1}^{n} k\left(d_{\mathfrak{C}}\left(\tilde{C}, \tilde{C}_{i}\right), \sigma\right),
$$

where $n$ is the number of curves, $d_{\mathfrak{C}}\left(\tilde{C}, \tilde{C}_{i}\right)$ is the distance metric between the curves $\tilde{C}$ and $\tilde{C}_{i}$ in the infinite dimensional space, $\mathfrak{C}$, and $k(x, \sigma)=N\left(x ; 0, \sigma^{2}\right)$. The kernel size $\sigma$ is computed from the variability of the level set functions [9].

We embed each curve $\tilde{C}_{i}$ by its corresponding level-set (signed distance) function $\psi_{\tilde{C}_{i}}$. Pixels with $\psi<0$ are inside the curve, where as pixels with $\psi>0$ are out of the curve. The magnitude of the signed distance function $\psi_{\tilde{C}_{i}}(x)$ is the shortest distance from the point $x$ to the curve $\tilde{C}_{i}$. Therefore, we define the distance between two curves, $\tilde{C}$ and $\tilde{C}_{i}$, as the distance between the two corresponding signed distance functions, $\psi_{\tilde{C}}$ and $\psi_{\tilde{C}_{i}}$. The distance metric is approximated by the $L^{2}$ distance (Euclidean norm) [9].

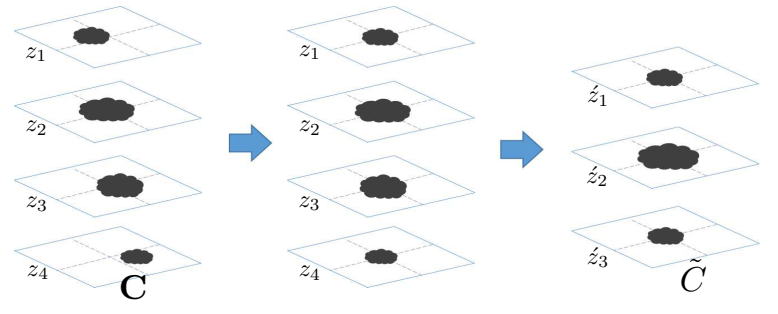

Fig. 3. Alignment of a $3 D$ nuclei image. The left image shows the unaligned image, where the curve $\mathbf{C}$ is the image boundary. We rotate and translate the image in 3 dimensions to obtain the aligned image (middle image). The centroid of the nuclei at each $2 D$ slice is at the center of the image and the orientation is zero in the $x y$ plane. As we fix the number of slices to 3 for our database, we perform polynomial interpolation as shown in the right image. This image boundary is $\tilde{C}$.
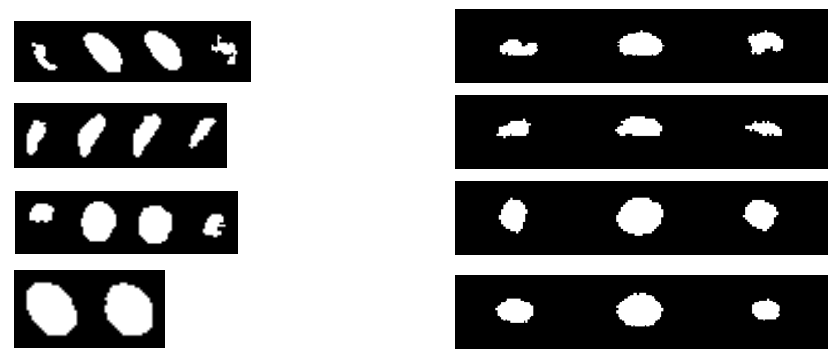

Fig. 4. Left: Selected training shapes: stacks of binary shape models of segmented nuclei selected interactively. These training shapes vary in the shapes, orientation, size and number of slices. Right: Alignment results of the training shapes. We standardized the number of image slices to three. Each training shape is scaled and translated in the $z$-direction such that the binary shape from the first and last slices are approximately half of the size of the middle slice.

\section{B. Alignment in $3 D$}

We develop a methodology to calculate the set of pose parameters $\left\{\mathbf{p}^{1}, \mathbf{p}^{2}, \ldots, \mathbf{p}^{n}\right\}$, in order to jointly align $n$ stacks of binary nuclei images. By aligning these images, we remove any variations in shape due to pose differences. In [6] and [9], alignment algorithms using gradient descent were applied to $2 D$ images. In [10], the same approach was applied to $3 \mathrm{D}$ magnetic resonance images. In that work, the number of image slices was fixed and variations in the $\mathrm{z}$ direction were minimal.

Our $3 D$ stacks of nuclei images were acquired with a resolution of $3 \mu \mathrm{m}$ in the z-direction and $0.6 \mu \mathrm{m}$ in the $x$ and $y$ planes. In these images, shown in Figure 4, there are nuclei with different poses (orientations, scale and coordinates) and different number of image slices. Some nuclei within the gel areas are more elongated in the $z$ direction compared to nuclei in non-gel areas.

We align $n 3 D$ nuclei images $I_{1: n}$ by firstly moving every pixel $(x, y, z)$ of image $I$ to a new position $T[\mathbf{p}](x, y, z)$ where

$$
T[\mathbf{p}]=R\left(\theta_{a b}, \theta_{a c}, \theta_{b c}\right)\left[\begin{array}{c}
h(x+a) \\
h(x+b) \\
h(x+c)
\end{array}\right] .
$$

Here $a, b$ and $c$ are the translation parameters, $\theta_{a b}, \theta_{a c}$ and $\theta_{b c}$ are the rotation angles for the $x y, x z$ and $y z$ planes respectively and $h$ is the scale factor. In an aligned image 




Fig. 5. A: Stack of images segmented by thresholding. B: Shape-marker based watershed algorithm to separate nuclei on each slice [4]. C: $3 D$ Watershed segmentation using methods in [5] and [2]. Each nuclei identified is assigned a different color

(e.g. the center image of Figure 3), the orientation of the nuclei to the $x y$ plane is zero and the centroid of the nuclei at each stack is aligned and centered around the middle of the image. The images were scaled to the mean nuclei size to reduce errors due to scaling.

Our $3 D$ nuclei images have different stacks sizes, ranging from 2 to 5 image slices as shown in the left column of Figure 4. We fix the number of image slices (stack size) for a training set. We chose to use 3 slices as aligned nuclei shapes are often unsymmetrical along the $z$ axis. As an example, the lower half of a nuclei is less elongated compared to its upper half if it is on a flat surface. We interpolate the image to obtain the nuclei shape near its top, at its middle and near its bottom.

We obtain the polynomial coefficients $\mathbf{P}$ over the existing nuclei size at each original. We select $z_{2}$ as the coordinate of the largest nuclei size while $\dot{z}_{1}$ and $\dot{z}_{3}$ the coordinates where the nuclei size is half of the maximum size at $z_{2}$. The relationship between $z$ coordinates of the original stack and the new stack is

$$
\left[\begin{array}{c}
\dot{z}_{1} \\
\vdots \\
\dot{z}_{n}
\end{array}\right]=\left[\begin{array}{cccc}
z_{1}^{3} & z_{1}^{2} & z_{1} & 1 \\
\vdots & \vdots & \vdots & \vdots \\
z_{n}^{3} & z_{n}^{2} & z_{n} & 1
\end{array}\right]\left[\begin{array}{c}
P_{3} \\
P_{2} \\
P_{1} \\
P_{0}
\end{array}\right]
$$

We interpolate in $3 D$ to obtain the aligned nuclei image slices at $\dot{z}_{1,2,3}$, as shown in Figure 3 .

Using $n=3$, the right column of Figure 4 shows the aligned and interpolated images of the corresponding images in the left column. This approach is less computationally expensive compared to storing a database of a large number of slices at small intervals. It is assumed that the size of the nuclei segmented is the largest in the middle of the stack of 2D slices and progressively becomes smaller towards to top and bottom of the stack. If there are multiple nuclei, this size variation assumption may not be valid and the interpolation will be poor. This is also a cause for nuclei misalignment.

\section{Alignment of clustered nuclei}

To construct our database, we assume the presence of only one underlying nucleus. If the input level set (image (B) in Figure 2) contains multiple nuclei, we would get inaccurate results if we evolve the level set with our prior

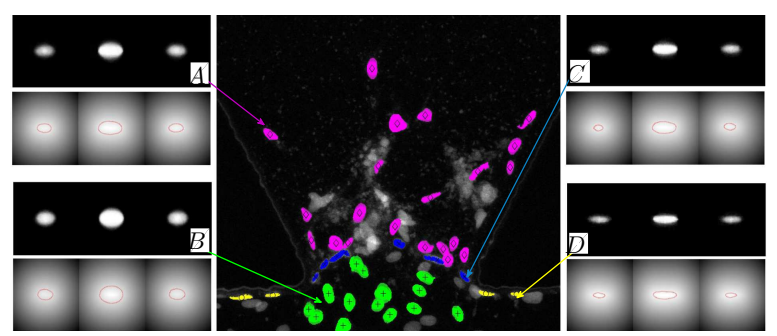

Fig. 6. Shape priors of different nuclei at different sections. (A) shows the mean shape and level set function for cells in the gel, (B), show the ones in areas with no gel. (C) shows the shape of the cells at the gel interface. These cells are more flatter in the $x y$ plane and longer in the $z$ plane. Likewise, the cells at the posts are flatter with less variation (shown in (D)).

model. Therefore, we propose to initially segment clustered nuclei using a watershed algorithm. We then estimate each separated nucleus shape and pose individually using shape priors.

We apply a shape-marker based watershed algorithm to split each $2 D$ slice of the image [4]. Classical watershed algorithms use the intensity region minima and ultimate eroded points as seed points. These approaches often result in oversegmentation. By using shape information to define markers, oversegmentation problems are reduced [4]. The second row in Figure 5 shows the results of applying the watershed algorithm separately on each $2 D$ slice. The left slice is the image on the bottom while the right most slice is the one on the top. In slice 3 , there are oversegmentation errors as a single nucleus is marked by 3 labels.

To correct the mistakes from the $2 D$ watershed algorithm, we apply a $3 D$ connection algorithm [5]. We create a set of nuclei labels with the $2 D$ watershed regions on the first slice. In the remaining image slices, processed from bottom to top, we compute the overlapping pixels between a watershed region with nuclei labeled regions on the lower slice. If the number of pixels exceed a minimum overlap, we group this region with its matching label on the lower slice. Otherwise, we assign a new nucleus label to that region.

If the watershed region overlaps more than one labeled region on its lower slice, we calculate the average fitting residual measure [2] and overlap amount. From those values, we either (a) assign the region to the larger labeled region, (b) group some or all of the lower overlapping regions together or (c) split the region. The third row of Figure 5 show that our $3 D$ connection algorithm successfully grouped the $2 D$ watershed labels. The separated watershed regions in slice 3 were grouped as well. We use the grouped watershed regions as the initial $3 D$ nuclei candidates and refine their curves by incorporating shape prior models.

The watershed algorithm requires $O(m+n \log n)$ operations. For an image of dimensions $X, Y$ by $Z, m=$ $X Y(Z-1)+X(Y-1) Z+(X-1) Y Z$ and $n=X Y Z$ [11]. The computational complexity of the level set algorithm is $O\left(p k^{2}\right)$ where $p$ is the number of points in the spatial direction and $k$ is the size of the narrow band which in our case, $k=5$ [12]. For each image, we run the watershed algorithm once and the level set algorithm multiple times. 


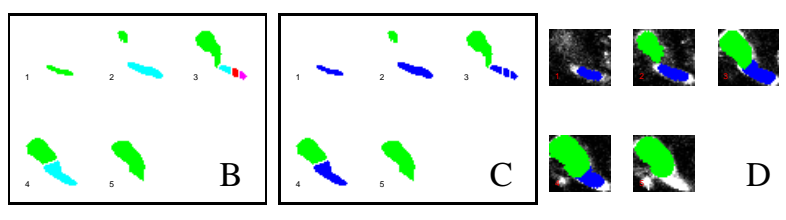

Fig. 7. Segmentation of two connected nuclei labeled blue and green. C: $3 D$ Watershed segmentation using methods in [5]. D : Improve shape estimate with a $3 D$ non-parametric shape prior.

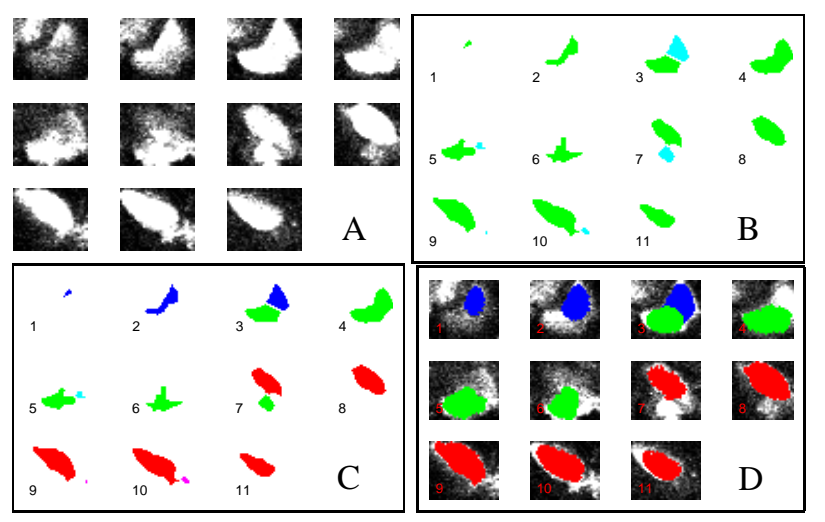

Fig. 8. Segmentation of three connected nuclei. Each segmented nuclei is labeled with a different color. A: Stack of original images acquired. B: Watershed algorithm on each slice [4]. C: $3 D$ connected components algorithm on watershed results [5]. D : Improved shape estimate by evolving the result with $3 D$ non-parametric shape priors.

\section{RESULTS}

We demonstrate the performance of our proposed method on real confocal microscopy images. The endothelial cells in microfludic devices [3] are stained with Hoechst and imaged with a 20x objective, with a resolution of 512 by 512 pixels. The image in Figure 6 shows that nuclei in different regions of the device are shaped differently. Therefore, we create and match to different shape databases for different nuclei coordinates.

We compare our methods with segmentation algorithms in [4] (Figures 7(B) and 8(B)) and [5] (Figures 7(C) and $8(\mathrm{C})$ ). By evolving the obtained curve with shape priors, we obtained more rounded and accurate nuclei shapes (Figures 7(D) and 8(D)). In Figure 8(D), the missing sections in slice 5 and 6 from the two other methods were filled in and the pixels in slice 4 do not overlap multiple nuclei. In comparison with work in [4], our method (shown in Figure 7(D)) overcomes oversegmentation issues and connects the nuclei over the 3D stack.

We confirm this observation with quantitative results in Table I. We segment the nuclei shapes manually and compare the results with our automated ones. We then compute the ratio of non-overlapping pixels over the total number of pixels. Figure 9 shows the $3 D$ reconstruction of the two examples.

\section{CONCLUSIONS}

We presented a new methodology for nuclei segmentation based on watershed and level sets. We initially segmented connected nuclei using watershed and refined their shape
TABLE I

\% OF PIXEL ERROR COMPARED WITH MANUAL RESULTS

\begin{tabular}{|c||c|c|}
\hline & $\begin{array}{c}\text { 2 Nuclei Dataset } \\
\text { (Figure 7) }\end{array}$ & $\begin{array}{c}\text { 3 Nuclei Dataset } \\
\text { (Figure 8) }\end{array}$ \\
\hline 3D Watershed [5] & $24.7 \%$ & $27.8 \%$ \\
Update with Shape Priors & $14.8 \%$ & $14.4 \%$ \\
\hline
\end{tabular}
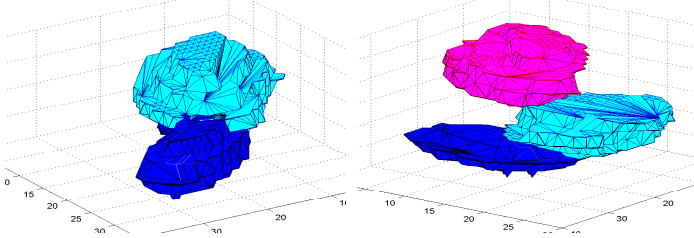

Fig. 9. $3 D$ reconstruction of the nuclei segmented. Left: 2 nuclei segmented in Figure 7. Right: 3 nuclei segmented in Figure 8.

estimates with prior models. We will extend our approach to segment $3 D$ cell cytoskeleton and track cell shapes from time-lapse confocal images.

\section{ACKNOWLEDGMENT}

This research was supported by the National Research Foundation Singapore through the Singapore MIT Alliance for Research and Technology's BioSystems and Micromechanics Inter-Disciplinary Research programme.

\section{REFERENCES}

[1] X. Bai, C. Sun and F. Zhou, Splitting touching cells based on concave points and ellipse fitting, in Pattern Recognition, vol. 42, 2009, pp. 2434-2446.

[2] C. Jung and C. Kim, Segmenting clustered nuclei using H-minima transform-based marker extraction and contour parameterization, in IEEE Trans Biomed Eng., vol. 57, no. 10, 2010, pp. 2600-2604.

[3] W. Farahat, L. Wood, I. Zervantonakis, A. Schor, S. Ong, D. Neal, R. Kamm and H. Asada, Ensemble Analysis of Angiogenic Growth in Three-Dimensional Microfluidic Cell Cultures, in PloS ONE, vol. 7, no. 5, 2012, pp. e37333.

[4] J. Cheng and J.C. Rajapakse, Segmentation of Clustered Nuclei With Shape Markers and Marking Function, in IEEE Trans. Biomed. Engineering, vol. 56, 2012, pp. 741-748.

[5] A. LaTorre, L. Alonso-Nanclares, S. Muelas, J.-M. Peña, and J. DeFelipe, 3D segmentations of neuronal nuclei from confocal microscope image stacks, in Front Neuroanat., vol. 7, 2013, pp. 1-10.

[6] A. Tsai, A. Yezzi, W. Wells, C. Tempany, D. Tucker, A. Fan, W. E. Grimson and A.Willsky, A shape-based approach to the segmentation of medical imagery using level sets, in IEEE Trans. Med. Imaging, vol. 22, 2003, pp. 137-154.

[7] T. F. Chan and L. A. Vese, Active contours without edges, in IEEE Trans. Image Proc., vol. 10, no. 2, 2001, pp. 266-277.

[8] T. Cootes, C. Taylor, D. Cooper, and J. Graham, Active shape models - their training and application, in Comput. Vis. Image Understanding, vol. 61,1995 , pp. 38-59.

[9] J. Kim, M. Çetin and A.S. Willsky, Nonparametric Shape Priors for Active Contour-based Image Segmentation, in Signal Processing, vol. 87, 2007, pp. 3021-3044.

[10] M.G. Uzunbaş, O. Soldea, D. Ünay, M. Çetin, G. Ünal, A. Erçil, A. Ekin, Coupled Nonparametric Shape and Moment-Based Intershape Pose Priors for Multiple Basal Ganglia Structure Segmentation in IEEE Trans. Med. Eng., vol. 29, no. 12, 2010, pp. 1959-1978.

[11] P. Felkel, M. Bruckschwaiger, R. Wegenkittl, Implementation and Complexity of the Watershed-from-Markers Algorithm Computed as a Minimal Cost Forest in Computer Graphics Forum, 2001, pp. 26-35.

[12] D. Adalsteinsson and J. A. Sethian, A Fast Level Set Method for Propagating Interfaces in Journal of Computational Physics, vol. 118, 1994, pp. 269-277. 